PREPARED FOR THE U.S. DEPARTMENT OF ENERGY, UNDER CONTRACT DE-AC02-76CH03073

PPPL-3862

PPPL-3862

UC-70

An Edge Rotation and Temperature Diagnostic on NSTX

by

T.M. Biewer, R.E. Bell, R. Feder, D.W. Johnson, and R.W. Palladino

August 2003

NM|

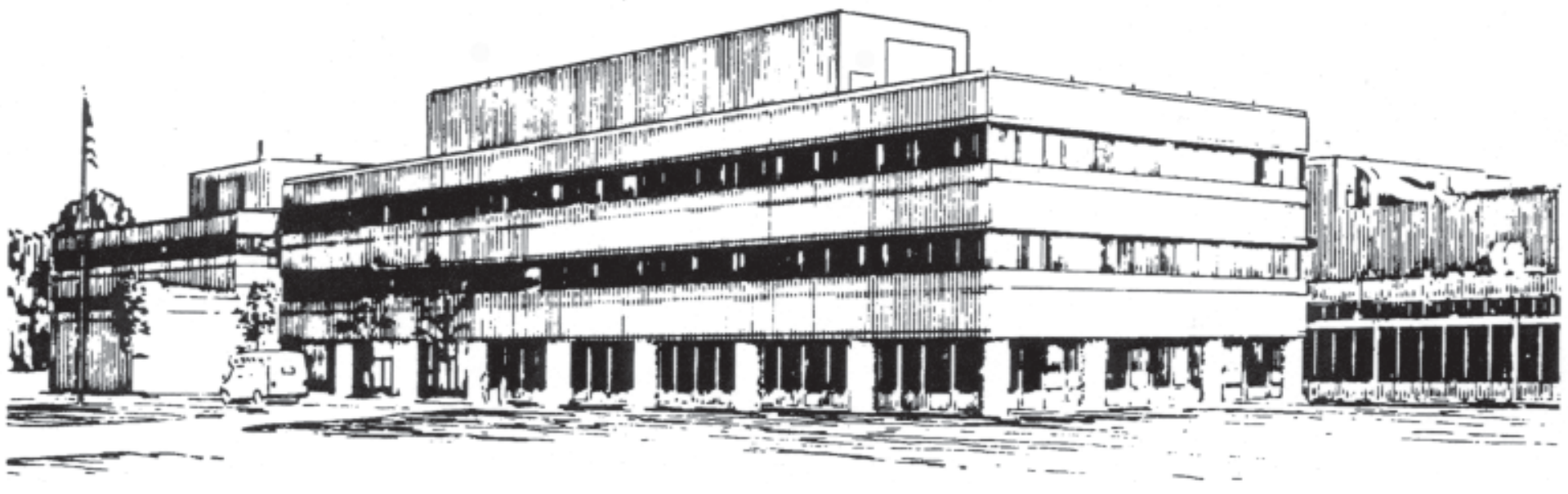

PRINCETON PLASMA PHYSICS LABORATORY PRINCETON UNIVERSITY, PRINCETON, NEW JERSEY 


\section{PPPL Reports Disclaimer}

This report was prepared as an account of work sponsored by an agency of the United States Government. Neither the United States Government nor any agency thereof, nor any of their employees, makes any warranty, express or implied, or assumes any legal liability or responsibility for the accuracy, completeness, or usefulness of any information, apparatus, product, or process disclosed, or represents that its use would not infringe privately owned rights. Reference herein to any specific commercial product, process, or service by trade name, trademark, manufacturer, or otherwise, does not necessarily constitute or imply its endorsement, recommendation, or favoring by the United States Government or any agency thereof. The views and opinions of authors expressed herein do not necessarily state or reflect those of the United States Government or any agency thereof.

\section{Availability}

This report is posted on the U.S. Department of Energy's Princeton Plasma Physics Laboratory Publications and Reports web site in Fiscal Year 2003. The home page for PPPL Reports and Publications is: http://www.pppl.gov/pub_report/

DOE and DOE Contractors can obtain copies of this report from:

U.S. Department of Energy

Office of Scientific and Technical Information

DOE Technical Information Services (DTIS)

P.O. Box 62

Oak Ridge, TN 37831

Telephone: (865) 576-8401

Fax: (865) 576-5728

Email: reports@adonis.osti.gov

This report is available to the general public from:

National Technical Information Service

U.S. Department of Commerce

5285 Port Royal Road

Springfield, VA 22161

Telephone: $1-800-553-6847$ or

(703) $605-6000$

Fax: (703) 321-8547

Internet: http://www.ntis.gov/ordering.htm 


\title{
An Edge Rotation and Temperature Diagnostic on NSTX
}

\author{
T.M. Biewer, R.E. Bell, R. Feder, D.W. Johnson, and R.W. Palladino \\ Princeton Plasma Physics Laboratory, Princeton, NJ 08543
}

(Dated: August 26, 2003)

\begin{abstract}
A new diagnostic for the National Spherical Torus Experiment (NSTX) is described whose function is to measure ion rotation and temperature at the plasma edge. The diagnostic is sensitive to $\mathrm{C}$ III, $\mathrm{C}$ IV, and He II intrinsic emission, covering a radial region of $15 \mathrm{~cm}$ at the extreme edge of the outboard midplane. Thirteen chords are distributed between toroidal and poloidal views, allowing the toroidal and poloidal rotation and temperature of the plasma edge to be simultaneously measured with $10 \mathrm{~ms}$ resolution. Combined with the local pressure gradient and EFIT reconstructed magnetic field profile, the edge flow gives a measure of the local radial electric field.
\end{abstract}

\section{INTRODUCTION}

Plasma rotation has been theoretically and experimentally linked with improved stability and confinement [14]. In particular, sufficient flow shear in the plasma edge is expected to suppress turbulent eddies and induce a transition from low- to high-confinement mode (L-mode to H-mode) as is observed in traditional tokamak magnetic confinement devices, such as DIII-D[5] and JET, and in spherical tokamaks[6] such as MAST[7] and NSTX[8]. It is believed that increased plasma flow is correlated with an increased radial electric field in the edge, leading to the L-to-H transition[9]. The radial electric field can be calculated from force balance, assuming that the toroidal and poloidal components of the plasma flow are measured, along with the magnetic field components and the plasma pressure. In this paper a new diagnostic is presented which measures the poloidal and toroidal flow velocities and temperatures in the edge of NSTX plasmas.

The intrinsic emission of edge ions can be extremely bright, and hence easily accessible as a plasma diagnostic. The intrinsic emission of $\mathrm{C}$ III impurity light, for example, rivals the brightness of the $\mathrm{D}_{\alpha}$ emission from deuterium fueled NSTX plasmas. Together, the emission from these lines constitutes the majority of visible light that is observable from typical NSTX discharges. Carbon is the main impurity in NSTX due to the presence of protective carbon tiles throughout the machine. Light is emitted by excited ions at the edge of the plasma primarily from electron impact ionization. Due to the presence of a steep electron temperature gradient at the edge, narrow shells of intrinsic emission are formed at the edge of the plasma. The intersection of a diagnostic sightline with an edge intrinsic emission shell gives a spatial localization to the spectroscopic measurement. Multiple sightlines tangent to the flux surfaces accommodate various plasma sizes, and with enough resolution can be inverted to give local measurements and gradients[10-12].

Because the intrinsic emission of the ions is used, active techniques to stimulate emission (e.g. neutral parti- cle beams) are not necessary, allowing measurements to be made in essentially all types of NSTX plasma discharges. Since this Edge Rotation Diagnostic (ERD) and the Charge Exchange Recombination Spectroscopy (CHERS)[13] diagnostic both measure the spectra of impurity ions, the techniques compliment each other, although CHERS measurements are limited to plasmas with neutral beam injected (NBI) power.

Since more than one spectral line is observed with the $\mathrm{ERD}$, it is possible to make simultaneous measurements of multiple ion species, in multiple charge states, and at multiple locations in the plasma. The diagnostic described here is sensitive to light from C III, C IV, and He II intrinsic emission.

\section{THE LIGHT DETECTION SYSTEM}

Light is collected from multiple sightlines through the plasma, as shown in Fig. 1 and Fig. 2, and focused onto a single CCD camera, after dispersion by a single spectrometer. Using a single spectrometer and CCD detector to image both poloidal and toroidal views saves cost and reduces systematic errors by simplifying calibration.

The collection optics are rigidly mounted outside of the vacuum vessel. The plasma is viewed poloidally through a $20 \mathrm{~cm}$ vacuum window on the top of the machine at Bay $\mathrm{B}$, which is located above a $5 \mathrm{~cm}$ gap in the NSTX passive stabilizing plates. Toroidally, the plasma is viewed through a $10 \mathrm{~cm}$ vacuum window off the pumping duct at the midplane of Bay L, which is shared by the CHERS background viewing optics. The CHERS background sightlines view toroidally at the midplane of the plasma, and the ERD sightlines are parallel to them, but displaced vertically downward by $\sim 2-3 \mathrm{~cm}$ at the radii of tangency. Shutters are mounted on the vacuum side of both windows for protection during vacuum vessel bakeout and He glow-discharge cleaning.

There are 6 poloidal and 7 toroidal sightlines, which utilize essentially identical optical systems to measure the light from intrinsic emission of the plasma. For the 


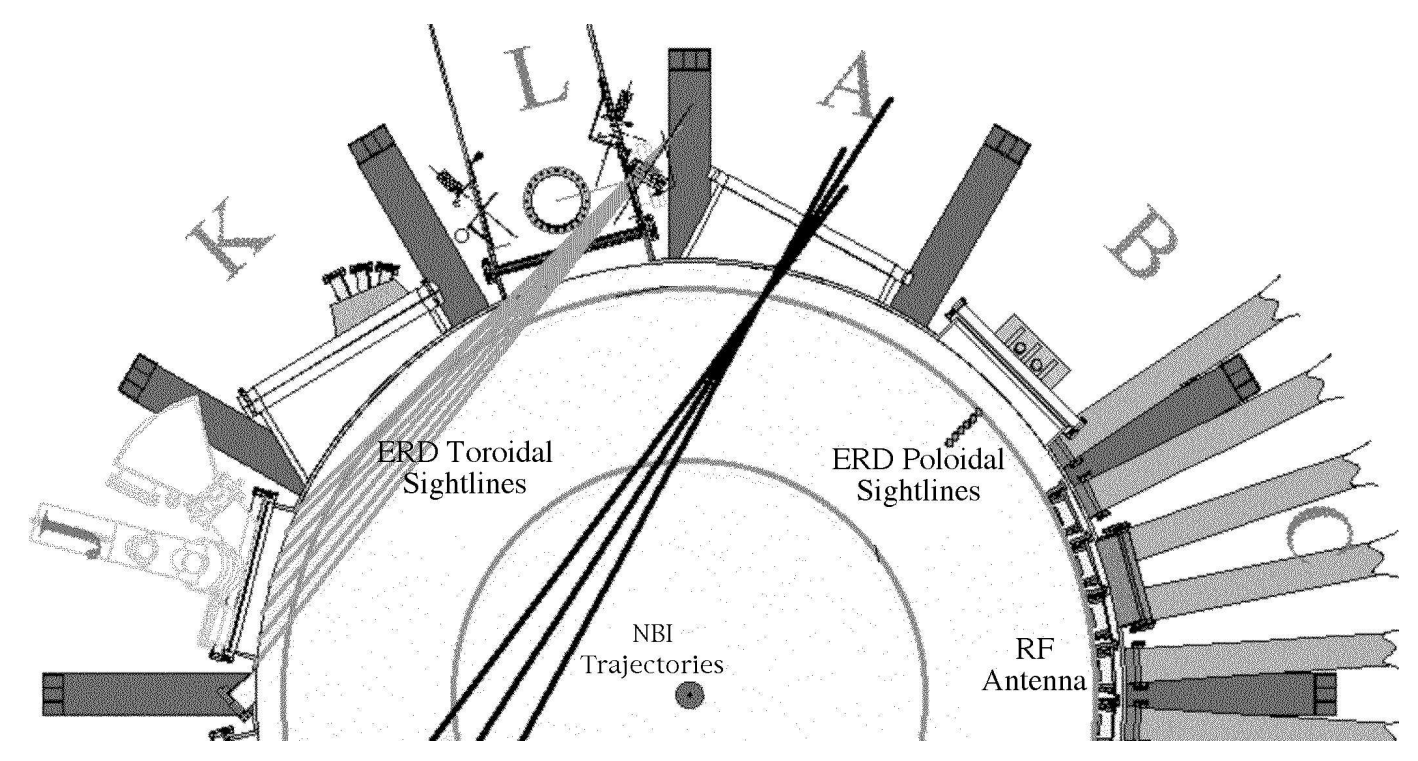

FIG. 1: Toroidal cross-section of the NSTX vacuum vessel showing the toroidal ERD sightlines.

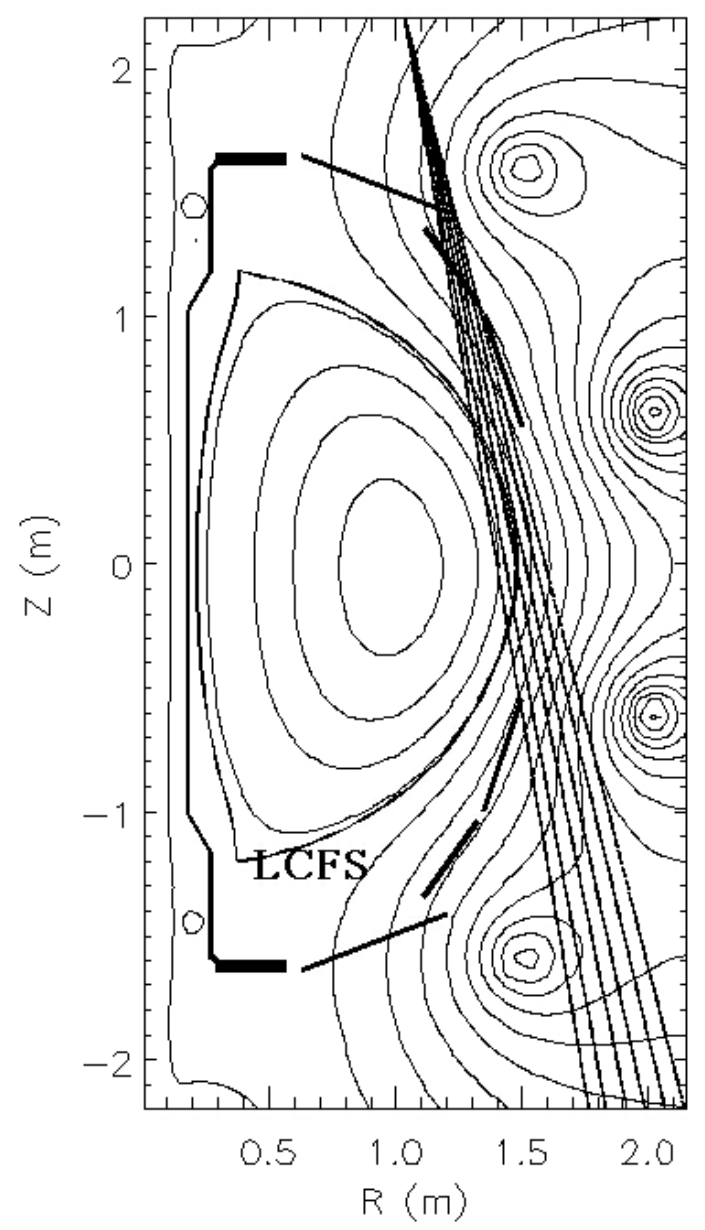

FIG. 2: Poloidal cross-section of the NSTX vacuum vessel showing the EFIT reconstruction of Shot 110077 at $313 \mathrm{~ms}$ and the poloidal ERD sightlines. poloidal sightlines, 6 fiber optics are held in a precisely machined mount at a fixed distance from a collection lens, and with an angular separation between fibers about the optical axis of the lens. This arrangement results in a fan of sightlines from the collection lens through the plasma. In the poloidal direction these sightlines are focused approximately $1.7 \mathrm{~m}$ from the collection lens, i.e. approximately on the midplane of the plasma. The images of the $600 \mu \mathrm{m}$ diameter fibers are $\sim 1.5 \mathrm{~cm}$ in diameter and spaced $3-4 \mathrm{~cm}$ apart on the midplane. In the toroidal direction, the sightlines are focused $1.7 \mathrm{~m}$ from the collection lens, i.e. at the point of tangency of the sightline to the plasma. The sightlines are parallel by design to the outermost sightlines of the CHERS background diagnostic, and utilize the same collection lens.

The light is collected from the plasma with two standard camera lenses (one toroidally and one poloidally viewing) of nominal $85 \mathrm{~mm}$ focal length at $f / 1.8$. These lenses are identical to the spectrometer input lens, giving optimal coupling between the collected plasma light and the spectrometer. The light is imaged from the plasma onto fiber optics, which carry the light from the NSTX machine area into an adjacent room, where the detection hardware is located. The output end of the 13 fibers are held vertically in a single column in front of the spectrometer entrance slit by a precisely machined mount.

The spectrometer is commercially available from Kaiser Optical Systems, Inc.[14] It is a modified Holospec Model HS-f/2.2-VIS, and uses a standard camera lens with a focal length of $85 \mathrm{~mm}$ at $f / 1.8$ to image the entrance slit. Light is focused through a high dispersion, holographic transmission grating that is sandwiched between two prisms. The center wavelength of the grating was selected to be $4650 \AA$, i.e. near the $\mathrm{C}$ in triplet. A $58 \mathrm{~mm}$ focal length output lens at $f / 1.2$ has been sub- 
stituted for the standard output lens. This lens arrangement demagnifies the image while preserving the etendue of the system.

Because of the short focal length of the spectrometer, there is a strong curvature of the image plane unless a curved entrance slit is used. A linear entrance slit would result in a strongly curved image. Hence, by imposing compensating curvature of the proper radius on the entrance slit, the image can be made linear. This effect is described in detail in Ref. [15]. The fiber holder was machined such that the fibers describe an arc with a radius of curvature of $39.22 \mathrm{~mm}$. This curvature is likewise imposed on the entrance slit of the spectrometer, which has a slit width of $75 \mu \mathrm{m}$ and a linear height of $11.3 \mathrm{~mm}$. The entrance slits were custom made by Lenox Laser[16].

The CCD detector was once commercially available from Roper Scientific[17] (Model Pentamax), and has a $512 \times 512$ array of $15 \times 15 \mu \mathrm{m}$ pixels, for an active area of $7.68 \times 7.68 \mathrm{~mm}$. The well depth of each pixel is $10^{5}$ electrons. The detector is thinned and back-illuminated, with a quantum efficiency of $68 \%$ at $4650 \AA$. The camera can read out at $5 \mathrm{MHz}$, with 12 bit resolution. With on-chip binning (47 bins), a single frame can be read out in $10 \mathrm{~ms}$. The camera is run by a dedicated PC, which is controlled by internal macro commands and external triggers, communicated via FTP. A 2-tab optical chopper wheel rotating at $50 \mathrm{~Hz}$ using Scitec Instruments[18] chopper-controler is used to shade the CCD during frame transfer, preventing image smearing. To accommodate the chopper between the spectrometer and camera bodies, it was necessary to replace the standard 4 inch chopper wheel with a balanced 6 inch wheel. The chopper is synchronized at $100 \mathrm{~Hz}$ to the NSTX master clock before the start of the discharge, along with the camera readout timing.

\section{RESULTS}

\section{Calibration}

Calibration of the ERD system requires multiple steps. The CCD camera must be aligned with the spectrometer. The spatial locations of the diagnostic sightlines must be measured. The channel-to-channel sensitivity of the sightlines must be taken into account. Finally, the spectral mapping of the CCD must be carefully calibrated.

The alignment of the CCD detector plane to the exit focal plane of the spectrometer is accomplished by micrometer adjustments to commercially available translation and rotation stages affixed to the spectrometer and camera bodies, which give 6 degrees-of-adjustment (3 translational and 3 rotational). This alignment was carried out with an illuminated slit before the fiber optics were attached to the spectrometer. A spare fiber mount was populated with two test fibers, and attached to the spectrometer. Filling these test fibers with white light yields two nominally horizontal bars of light on the detector. The rotation of the camera with respect to the spectrometer was adjusted until the two lines of white light were alligned with the CCD rows. Replacing the white light source with a neon gas filled, copper hollow-cathode lamp, gives a spectrum of lines, which can be used to adjust the translational and other rotational degrees of freedom. The width of the bright $\mathrm{Cu} 4651 \AA$ line was used to adjust the focus of the spectrometer. Fine adjustments to these settings were later made after the fiber optic bundle was attached to the spectrometer, using the copper hollow-cathode lamp to fill the input end of the fibers. A neon pen-lamp was occasionally used throughout this process, in place of the $\mathrm{Cu}$ hollow-cathode lamp. There are many neon lines within the spectral range of this spectrometer, which were helpful in checking the alignment of different regions of the CCD detector, though the neon lines are considerably weaker than the $\mathrm{Cu} 4651 \AA$ line.

The spatial locations of the diagnostic sightlines were found through an in-vessel calibration process with backlit fibers. A bright, white light source was projected onto the output end of the fibers. By placing a screen inside the vacuum vessel, the projection of light indicated where each fiber was viewing. The position of the back-lit spot was measured at multiple positions along the sightline (by moving the screen), with respect to the wall of the vessel through the use of a calibrated FARO Measurement Arm[19]. Fitting a line through the spot locations of each fiber, gave an accurate measure of the sightline path. This was done for both the poloidal and toroidal views.

After the fibers were attached to the spectrometer, spectral calibrations could be completed with the entire optical system in its final configuration. Minor adjustments to the camera/spectrometer orientation were made, as discussed above. Then, from inside the vacuum vessel, an extended, uniform white light source was used to fill the collection optics of first the poloidal, then the toroidal views. This provided a channel-to-channel, relative sensitivity calibration of the detector, which is referred to as a "white plate" calibration. The white plate was cross-calibrated with a Labsphere[20] blackbody source to get an absolute intensity calibration.

It is important when measuring the instrument transfer function to fill the optics in the same manner as for the experimental measurements. The preferred technique was to use the light emitted by a vessel-filling, neon glowdischarge. This method has the advantage that the calibration is performed with light that is gathered along the exact sightlines of the diagnostic that measurements of rotation and temperature are made during plasma shots. A parallel technique done in vessel uses the copper hollow cathode lamp with a collimating lens to individually fill each fiber. The $\mathrm{Cu} 4651 \AA$ line and minor neon lines were used to get the spectral calibration of each detector 
channel. The dispersion of the system is roughly 0.222 $\AA /$ pixel, though it varies somewhat across the CCD. The instrumental temperature is $\sim 40 \mathrm{eV}$, which corresponds to an instrumental width of $\sim 2.9$ pixels.

\section{Operation}

Since this was a new, prototype diagnostic, some adjustments to the diagnostic were necessary once initial measurements were made. In the original configuration the ERD suffered from saturated channels. Adding additional CCD readout bins (i.e. less light per bin) to the brightest sightlines, mitigated some of the problem. Maintaining a $10 \mathrm{~ms}$ frame rate imposed a 47 bin maximum. The aperture of the spectrometer entrance lens had to be decreased to limit the light intensity on the CCD. Ultimately, an aperture of $f / 11$ was deemed necessary, which collects only $2.7 \%$ of the light available at $f / 1.8$. Even at this reduced aperture, the ERD measures $>10^{5}$ photoelectrons of $\mathrm{C}$ III light in $10 \mathrm{~ms}$ for a given sightline during typical operating conditions. The light collection system described here was originally designed for the poloidal rotation system on the Tokamak Fusion Test Reactor (TFTR)[15], which required high throughput, and was later adapted for use on NSTX. Clearly, the present application would benefit greatly from a faster CCD camera, allowing higher time resolution data to be measured without sacrificing the present signal-to-noise ratio (by opening the aperture).

\section{Experimental Data}

The NSTX is a large spherical tokamak with a major radius of $0.85 \mathrm{~m}$ and a minor radius of $0.65 \mathrm{~m}$. The plasma poloidal cross-section is strongly shaped with high vertical elongation, as can be seen in Fig. 2. Typical pulse lengths of NSTX discharges are $\simeq 1 \mathrm{~s}$, with an onaxis toroidal magnetic field between 0.3 and $0.6 \mathrm{~T}$. The plasma current is typically $\leq 1.5 \mathrm{MA}$.

Fig. 3 shows sample spectra from two NSTX discharges. A full frame of data covers the wavelength range from 4595 to $4705 \AA$. Partial spectra are shown here to highlight the C III, C IV, and He II lines. The error bars represent the statistical error in the data. The error is small, due to the large number of detected photons. The C III triplet $(4647.419,4650.246$, and $4651.473 \AA$ ) is from the ${ }^{3} S$ to ${ }^{3} P_{0}$ transition[21]. The relative amplitude and wavelengths of the three lines are well known. A three Gaussian fit to the data is constrained by the relative amplitudes and the fixed relative separations of the three spectral lines. Thus, the three free parameters which are fit to the data are the overall amplitude, the line shift of the triplet (the plasma velocity), and a single line width (the ion temperature). Fitting to all three lines simulta-
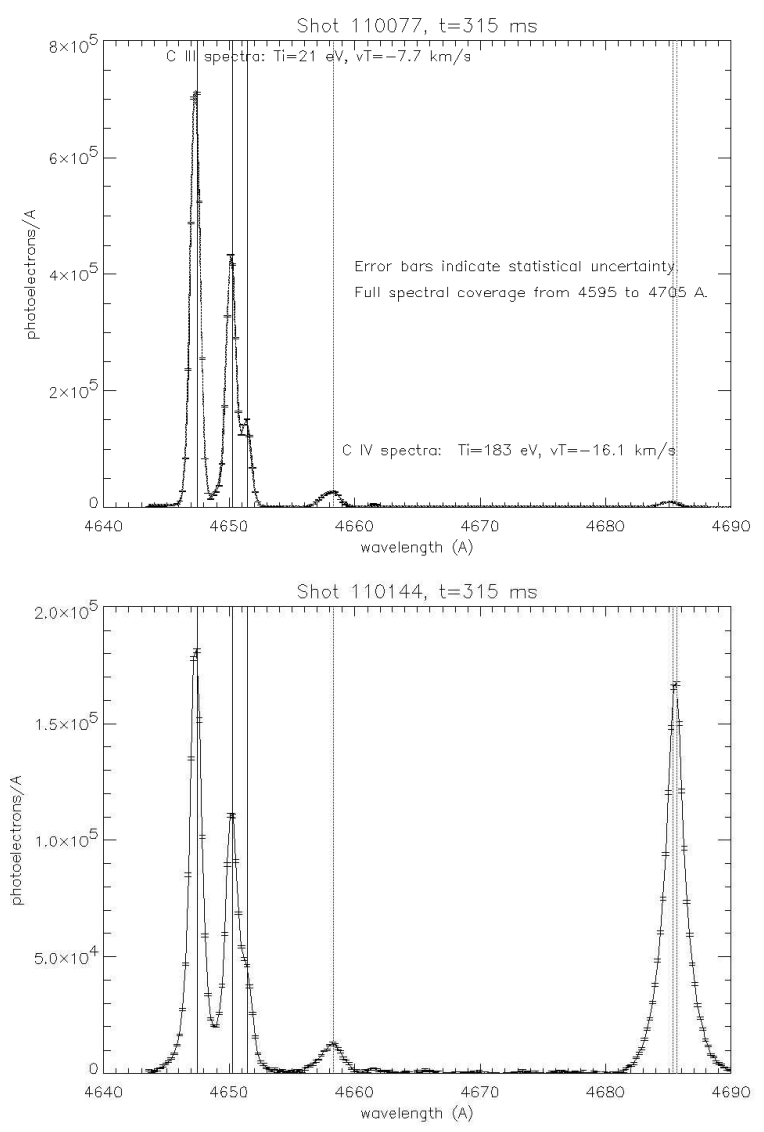

FIG. 3: Sample spectra from (a) Shot 110077 (NBI heated $\mathrm{D}_{2}$ plasma) and (b) Shot 110144 (RF heated He plasma). The non-shifted line centers are represented by the vertical solid lines ( $\mathrm{C}$ III), dashed lines ( $\mathrm{C}$ IV), and dotted line (He II). Error bars indicate the statistical uncertainty in the data.

neously not only increases the amount of signal utilized, but also improves the robustness of the fit, since parasitic lines (i.e. non $\mathrm{C}$ III) within the fit range are unlikely to effect the fit.

Each sightline gives a line-averaged measure of the intrinsic emission of a given species in the edge of the plasma. By inverting the data, a local measure of the ion spectral line emissivity, velocity, and temperature can be found. Fig. 4(a) shows typical edge profiles of the C III brightness as measured by the toroidal and poloidal views during Shot 110077. These data can be inverted to give the local emissivity of the C III shell[10-12], as shown in Fig. 4(b). In the toroidal view, the radii listed are the tangency radii of the sightlines. Since the poloidal crosssection is non-circular, the radii listed for the poloidal view are the midplane radii of the flux surface where each sightline is tangent (above the midplane.) Also shown in Fig. 4 is the radial location of the last closed flux surface (LCFS) as calculated from the EFIT equilibrium reconstruction[22], which occurs just outside the peak in 


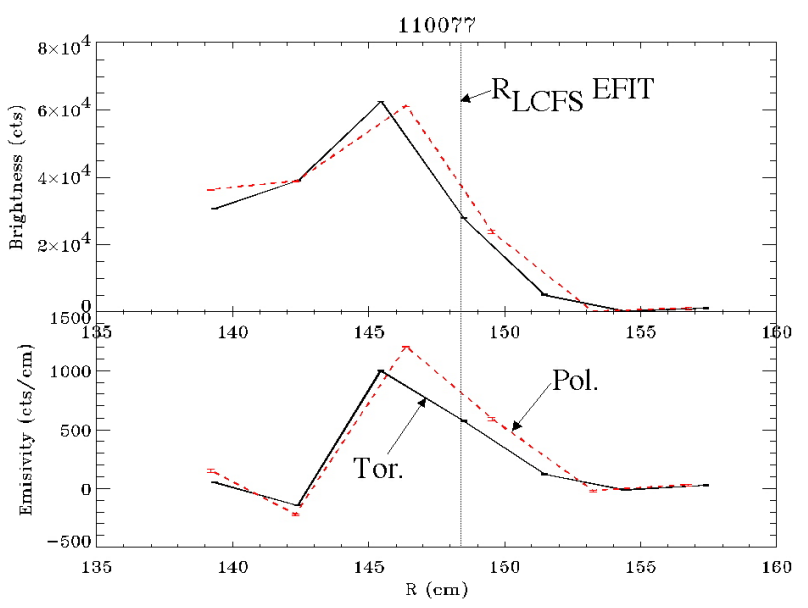

FIG. 4: Edge profiles of C III (a) brightness and (b) emissivity from the poloidal (dashed) and toroidal (solid) views at $315 \mathrm{~ms}$ in Shot 110077.

the $\mathrm{C}$ III emission. The agreement between the toroidal and poloidal views (which are toroidally separated by 90 degrees) in the amplitude and location of the emission shell is indicative that the intrinsic emission is isotropic on the flux surfaces.

The radial width of the emission shell in this discharge is on the order of the sightline separation. Hence, the edge ion velocity (and temperature) can only be measured for C III at one (or possibly two) radial locations. Observations show that the radius of peak emissivity evolves during discharges over the radial range of the diagnostic. Thus, while the radial coverage of the ERD appears apt for a measurement of the local velocity (and hence radial electric field), the radial resolution may be inadequate to measure gradients in these quantities for a single charge state in a discharge with a steep electron temperature gradient. The radial resolution of the ERD could be improved by replacing the $600 \mu \mathrm{m}$ diameter fibers with a more closely spaced array of $200 \mu \mathrm{m}$ diameter fibers. This factor of 10 reduction in gathered light could easily be countered by increasing the aperture of the optics from $f / 11$.

Shot 110077 is a double-null, $\mathrm{D}_{2}$ fueled discharge, with a plasma current of $1 \mathrm{MA}$, on-axis toroidal magnetic field of $0.45 \mathrm{~T}$, and up to $5.1 \mathrm{MW}$ of neutral beam injected (NBI) power. The discharge transits into "high confinement mode," or H-mode beginning around $240 \mathrm{~ms}$ and lasts for roughly $250 \mathrm{~ms}$, until the discharge terminates. During the H-mode period, there are multiple bursts of edge-localized modes (ELMs), indicated by spikes in the $\mathrm{D}_{\alpha}$ trace shown in Fig. 5(a). A closer inspection reveals that there are also ELM-free H-mode periods, e.g. from 230 to $250 \mathrm{~ms}$, and from 305 to $330 \mathrm{~ms}$. The NBI power is increased stepwise by $1.7 \mathrm{MW}$ at 60,160 , and $320 \mathrm{~ms}$ from 0 to $5.1 \mathrm{MW}$. Fig. 5(b) shows the time evolution of

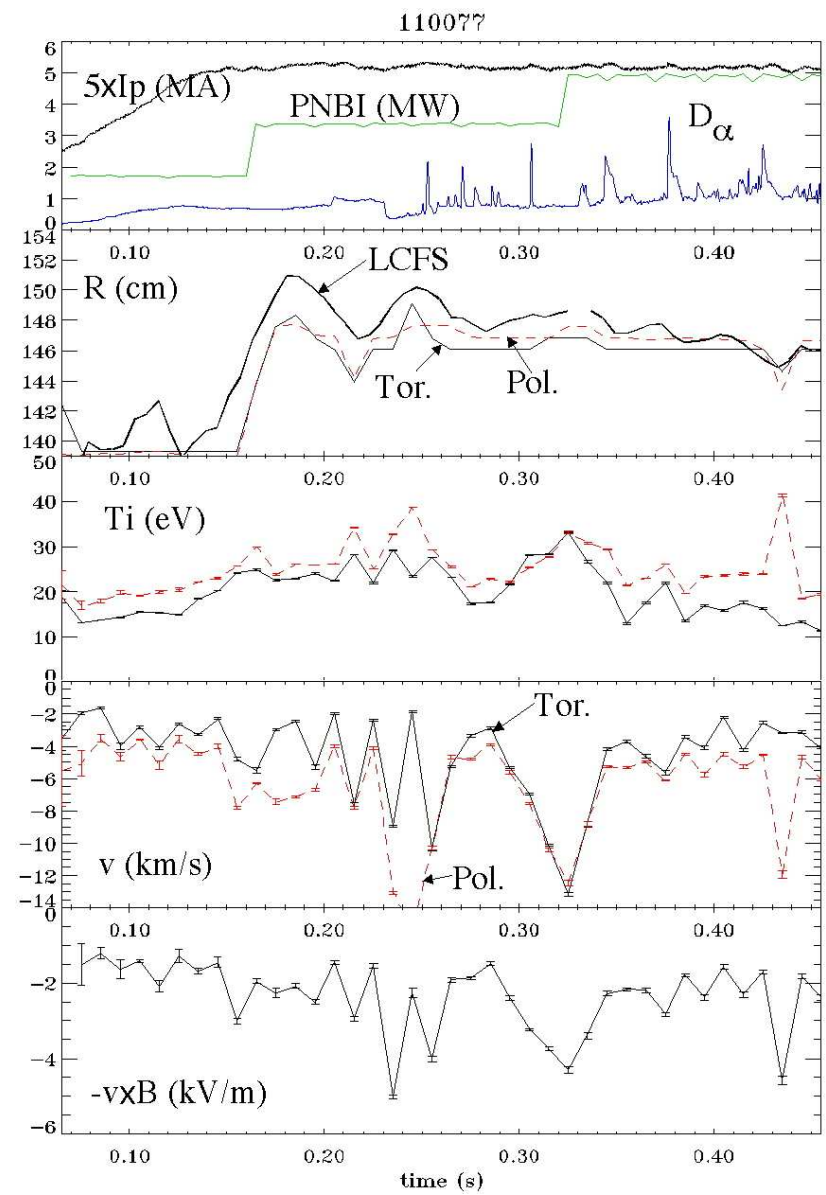

FIG. 5: Sample data during Shot 110077, showing (a) the plasma current, neutral beam injected power, and a $\mathrm{D}_{\alpha}$ trace, and from the $\mathrm{C}$ III lines (solid=toroidal, dashed=poloidal): (b) radii of peak emissivity compared to EFIT calculated LCFS (heavy solid), (c) the apparent temperature at the above radii, (d) and the local rotation velocity. (e) $E_{r} \sim-(\vec{v} \times \vec{B})_{r}$ from the above measurements.

the radii of peak toroidal and poloidal $\mathrm{C}$ III emission as the discharge progresses, along with the EFIT calculated location of the LCFS. Fig. 5(c) and (d) show the time evolution of the apparent temperature and local velocity, respectively, for both the toroidal and poloidal views at the radii of maximum emissivity. The error bars in the plots are indicative of the propagated statistical error of the measurement. Toroidally, a positive velocity is in the direction of the plasma current and the neutral beams. Poloidally, a positive velocity is downward on the outboard side of the machine.

From force balance $E_{r}=-(\vec{v} \times \vec{B})_{r}+\nabla p / Z e n$, for each species. Fig. $5(\mathrm{f})$ shows the contribution to the radial electric field, $E_{r}$, from the radial $-\vec{v} \times \vec{B}$ terms, calculated from these measurements and the EFIT reconstruction of the magnetic field, $\vec{B}$. If the $\nabla p / Z e n$ term is small, as it 
should be at the peak of the emissivity, then this term is a good estimation of the local radial electric field.

\section{SUMMARY}

A new diagnostic is described that measures the dynamics of ions (C III, C IV, and He II ) in the plasma edge. The ERD simultaneously measures poloidal and toroidal rotation in the edge plasma. This facilitates a passive, i.e. non-invasive, estimation of the edge radial electric field. Since the ERD relies on intrinsic emission of edge ions, it can be used in all discharges. These measurements are made with $10 \mathrm{~ms}$ resolution, limited by the CCD detector read-out rate. At this rate, $97.3 \%$ of the available emission light must be removed by reducing the aperture to prevent saturation of the CCD detector. A clear path to improving the diagnostic would be to replace the current $\mathrm{CCD}$ with a faster model, which is presently available commercially. This would yield submillisecond time resolution of the poloidal and toroidal edge ion dynamics, without sacrificing the data quality already obtained. The radial resolution of the ERD could further be improved by adding more, smaller fiber optics. This would provide improved localization of the peak of the emission shell with multiple measurements across a given emission shell, allowing local gradients to be more accurately measured.

\section{ACKNOWLEDGMENTS}

The authors wish to recognize the many contributions of the NSTX group, particularly Steve Sabbagh for providing the EFIT reconstructions. This research was supported by the U.S. D.O.E. under contract: DE-AC0276CH03073.

[1] W. M. Stacey and M. Murakami, Phys. Plasmas 8, 4450 (2001).

[2] W. M. Stacey and J. Mandrekas, Phys. Plasmas 9, 1622 (2002).
[3] K. Ida, Plasma Physics of Controlled Fusion 40, 1429 (1998).

[4] K. Ida, S. Hidekuma, M. Kojima, Y. Miura, S. Tsuji, K. Hoshino, M. Mori, N. Suzuki, and T. Yamauchi, Phys. Fluids B 4, 2552 (1992).

[5] T. N. Carlstrom, R. J. Groebner, C. Fenzi, G. R. McKee, R. A. Moyer, and T. L. Rhodes, Plasma Physics of Controlled Fusion 44, 333 (2002).

[6] Y.-K. Peng and D. Strickler, Nuclear Fusion 26, 769 (1986).

[7] A. Sykes, J. W. Ahn, R. Akers, E. Arends, P. G. Carolan, G. F. Counsell, S. J. Fielding, M. Gryaznevich, R. Martin, M. Price, et al., Phys. Plasmas 8, 2101 (2001).

[8] J. Spitzer, M. Ono, M. Peng, D. Bashore, T. Bigelow, A. Brooks, J. Chrzanowski, H. M. Fan, P. Heitzenroeder, T. Jarboe, et al., Fusion Technology 30, 1337 (1996).

[9] R. J. Akers, G. F. Counsell, A. Sykes, L. C. Appel, E. R. Arends, C. Byrom, P. G. Carolan, N. J. Conway, G. Cunningham, A. Dnestrovskij, et al., Phys. Rev. Lett. 88, 1 (2002).

[10] R. E. Bell, Rev. Sci. Instrum. 66, 558 (1994).

[11] R. E. Bell, Rev. Sci. Instrum. 68, 1273 (1996).

[12] I. Condrea, E. Haddad, B. C. Gregory, and G. Abel, Phys. Plasmas 7, 3641 (2000).

[13] B. Stratton, R. J. Fonk, K. P. Jaehnig, N. Schechtman, and E. J. Synakowski, in Proceedings of the IAEA Technical Committee Meeting on Time Resolved Two- and Three-Dimensional Plasma Diagnostics, Najoya, Japan, 1990 (IAEA, Vienna, 1991), p. 78.

[14] Kaiser Optical Systems, Inc., Ann Arbor, Michigan, http : //www.kosi.com.

[15] R. E. Bell, L. E. Dudek, B. Grek, D. W. Johnson, and R. W. Palladino, Rev. Sci. Instrum. 70, 821 (1998).

[16] Lenox Laser, Inc., Glen Arm, Maryland, http : //www.lenoxlaser.com.

[17] Roper Scientific, Inc., Trenton, New Jersey, http : //www.roperscientific.com.

[18] Scitec Instruments, Ltd., Cornwall, England, http : //www.scitec.uk.com.

[19] FARO Technologies, Inc., Lake Mary, Florida, http : //www.faro.com.

[20] Labsphere Inc., Sutton, New Hampshire, http : //www.labsphere.com.

[21] A. R. Striganov and N. S. Sventitskii, Tables of Spectral Lines of Neutral and Ionized Atoms. (Plenum Press, 1968).

[22] L. L. Lao, H. S. John, R. D. Stambaugh, A. G. Kellman, and W. Pfeiffer, Nuclear Fusion 25, 1611 (1985). 


\section{External Distribution}

Plasma Research Laboratory, Australian National University, Australia

Professor I.R. Jones, Flinders University, Australia

Professor João Canalle, Instituto de Fisica DEQ/IF - UERJ, Brazil

Mr. Gerson O. Ludwig, Instituto Nacional de Pesquisas, Brazil

Dr. P.H. Sakanaka, Instituto Fisica, Brazil

The Librarian, Culham Laboratory, England

Mrs. S.A. Hutchinson, JET Library, England

Professor M.N. Bussac, Ecole Polytechnique, France

Librarian, Max-Planck-Institut für Plasmaphysik, Germany

Jolan Moldvai, Reports Library, Hungarian Academy of Sciences, Central Research Institute for Physics, Hungary

Dr. P. Kaw, Institute for Plasma Research, India

Ms. P.J. Pathak, Librarian, Institute for Plasma Research, India

Ms. Clelia De Palo, Associazione EURATOM-ENEA, Italy

Dr. G. Grosso, Instituto di Fisica del Plasma, Italy

Librarian, Naka Fusion Research Establishment, JAERI, Japan

Library, Laboratory for Complex Energy Processes, Institute for Advanced Study, Kyoto University, Japan

Research Information Center, National Institute for Fusion Science, Japan

Dr. O. Mitarai, Kyushu Tokai University, Japan

Dr. Jiangang Li, Institute of Plasma Physics, Chinese Academy of Sciences, People's Republic of China

Professor Yuping Huo, School of Physical Science and Technology, People's Republic of China

Library, Academia Sinica, Institute of Plasma Physics, People's Republic of China

Librarian, Institute of Physics, Chinese Academy of Sciences, People's Republic of China

Dr. S. Mirnov, TRINITI, Troitsk, Russian Federation, Russia

Dr. V.S. Strelkov, Kurchatov Institute, Russian Federation, Russia

Professor Peter Lukac, Katedra Fyziky Plazmy MFF UK, Mlynska dolina F-2, Komenskeho Univerzita, SK-842 15 Bratislava, Slovakia

Dr. G.S. Lee, Korea Basic Science Institute, South Korea

Institute for Plasma Research, University of Maryland, USA

Librarian, Fusion Energy Division, Oak Ridge National Laboratory, USA

Librarian, Institute of Fusion Studies, University of Texas, USA

Librarian, Magnetic Fusion Program, Lawrence Livermore National Laboratory, USA

Library, General Atomics, USA

Plasma Physics Group, Fusion Energy Research Program, University of California at San Diego, USA

Plasma Physics Library, Columbia University, USA

Alkesh Punjabi, Center for Fusion Research and Training, Hampton University, USA

Dr. W.M. Stacey, Fusion Research Center, Georgia Institute of Technology, USA

Dr. John Willis, U.S. Department of Energy, Office of Fusion Energy Sciences, USA

Mr. Paul H. Wright, Indianapolis, Indiana, USA 
The Princeton Plasma Physics Laboratory is operated by Princeton University under contract with the U.S. Department of Energy.

\author{
Information Services \\ Princeton Plasma Physics Laboratory \\ P.O. Box 451 \\ Princeton, NJ 08543
}

Phone: 609-243-2750

Fax: 609-243-2751

e-mail: pppl_info@pppl.gov

Internet Address: http://www.pppl.gov 